\title{
Gassensor zur Konzentrationsüberwachung von Methan bei Wärmebehandlungsprozessen
}

\author{
S. Schröder, H. Fritze \\ TU Clausthal, Institut für Energieforschung und Physikalische Technologien, \\ Am Stollen 19 B, 38640 Goslar, Deutschland \\ sebastian.schroeder@tu-clausthal.de
}

\begin{abstract}
Zusammenfassung
Die Überwachung der Ofenatmosphäre bei Wärmebehandlungsprozessen ermöglicht es, direkte Rückschlüsse auf den Bauteilzustand im Ofen zu ziehen. Daher wird im Verbundvorhaben „Konzentrationsabhängige Industrieofen-Regelung" eine Technologie entwickelt, bei der die Messung der Ofenatmosphäre eine energieeffiziente Regelung von elektrisch beheizten Wärmebehandlungsprozessen ermöglicht. Eine solche Technologie bietet ein erhebliches Energieeinsparpotential. Die Überwachung der Ofenatmosphäre kann dabei abhängig vom konkreten Materialsystem auf eine überschaubare Anzahl von Schlüsselgasspezies beschränkt werden. Ein Beispiel bildet Methan, das bei Temperaturen um $700{ }^{\circ} \mathrm{C}$ detektiert werden muss. Zur Messung der Methankonzentration wird ein Gassensorprinzip umgesetzt, das die Selektivität metalloxidbasierter Gassensoren erhöht, in dem neben den elektrischen auch mechanische Eigenschaften der Sensorschicht mit Hilfe hochtemperaturstabiler piezoelektrischer Resonatoren bestimmt werden. Als Sensorschicht wird z. B. Praseodym dotiertes Ceroxid $\mathrm{Pr}_{0,1} \mathrm{Ce}_{0,9} \mathrm{O}_{2-\delta}$ verwendet, das auf einkristalline $\mathrm{Ca}_{3} \mathrm{TaGa}_{3} \mathrm{Si}_{2} \mathrm{O}_{14}$ Resonatoren aufgebracht wird.
\end{abstract}

Keywords: Gassensoren, Metalloxid, piezoelektrische Resonatoren, Dünne Schichten, Praseodym dotiertes Ceroxid

\section{Motivation}

Die bei Wärmebehandlungsprozessen zu erwartenden Temperaturen und Gasatmosphären erfordern den Einsatz hochtemperaturstabiler Gassensoren, die oxidierenden und reduzierenden Atmosphären widerstehen können. Der Einsatz optischer Sensoren ist dabei aufgrund einer erschwerten Systemintegration und hoher Kosten für den regulären Betrieb nicht vorgesehen. Resistive Metalloxidgassensoren wären prinzipiell für den Anwendungszweck geeignet. Sie weisen jedoch eine zu geringe Gasselektivität auf, um den Konzentrationsverlauf prozessspezifischer Schlüsselgasspezies überwachen zu können. Aus diesem Grund soll ein Sensorprinzip zum Einsatz kommen, welches die Selektivität metalloxidbasierter Gassensoren erhöht, indem neben den elektrischen auch mechanische Eigenschaften der Sensorschicht mit Hilfe von hochtemperaturstabilen piezoelektrischen Resonatoren bestimmt werden.

Als piezoelektrisches Resonatormaterial finden einkristalline $\mathrm{Ca}_{3} \mathrm{TaGa}_{5} \mathrm{Si}_{2} \mathrm{O}_{14} \quad$ (CTGS, $y$-Schnitt) Substrate Anwendung. Diese werden als Mikrowaage betrieben und zu Dicken- scherschwingungen angeregt. CTGS zeichnet sich durch eine hohe Güte der Resonanz aus [2] und ist in reduzierenden Atmosphären bis zu einem Sauerstoffpartialdruck von $10^{-15}$ bar bis $1000{ }^{\circ} \mathrm{C}$ stabil.

\section{Defektchemie von $\operatorname{Pr}_{\mathrm{x}} \mathrm{Ce}_{1-\mathrm{x}} \mathrm{O}_{2-\delta}$}

Die Defektchemie von $\mathrm{Pr}_{x} \mathrm{Ce}_{1-\mathrm{x}} \mathrm{O}_{2-\delta}$ (PCO) kann mit einem Model beschrieben werden, wie es z. B. in [3] vorgestellt wird. Die Einlagerung bzw. der Ausbau von Sauerstoffionen aus dem Gitter wird abhängig vom Sauerstoffpartialdruck durch die Oxidationspaare $\mathrm{Pr}^{3+} / \mathrm{Pr}^{4+}$ und $\mathrm{Ce}^{3+} / \mathrm{Ce}^{4+}$ elektrisch kompensiert. Für Sauerstoffpartialdrücke oberhalb von ca. $10^{-}$ ${ }^{5}$ bar kompensieren Pr-Ionen den Ausbau der Sauerstoffionen, in dem sie von $\mathrm{Pr}^{4+} \mathrm{zu} \mathrm{Pr}^{3+}$ reduziert werden. Gl. (1) beschreibt das Defektgleichgewicht in Kröger-Vink-Notation.

$\operatorname{Pr}_{\mathrm{Ce}}^{\mathrm{x}}+\mathrm{O}_{\mathrm{O}}^{\mathrm{x}} \rightleftharpoons 2 \mathrm{Pr}_{\mathrm{Ce}}^{\prime}+\mathrm{V}_{\mathrm{O}}^{\prime \prime}+1 / 2 \mathrm{O}_{2(\mathrm{~g})}$

Dabei stehen $\operatorname{Pr}_{\mathrm{Ce}}^{\mathrm{x}}, \mathrm{Pr}_{\mathrm{Ce}}^{\prime}, \mathrm{O}_{0}^{\mathrm{x}}$ und $\mathrm{V}_{0}$ 仙 $\operatorname{Pr}^{4+}$, $\mathrm{Pr}^{3+}$, Sauerstoffionen und Sauerstoffleerstellen. Aus der vereinfachten Neutralitätsbedingung $2\left[\mathrm{Pr}_{\mathrm{Ce}}^{\prime}\right] \approx\left[\mathrm{V}_{\mathrm{O}}^{\prime \prime}\right]$ ergibt sich der Zusammen- 
hang von Sauerstoffleerstellenkonzentration und Sauerstoffpartialdruck (siehe GI. (2)).

$\left[\mathrm{V}_{\mathrm{O}}\right] \sim \mathrm{p}_{\mathrm{O}_{2}}^{-1 / 6}$

Sind alle Pr-Ionen reduziert, wird die Sauerstoffleerstellenkonzentration unter Einhaltung der Neutralitätsbedingung fixiert. Erst bei sehr niedrigen Sauerstoffpartialdrücken von ca. $10^{-15}$ bar können bei $700{ }^{\circ} \mathrm{C}$ durch die Reduktion der Ce-lonen weitere Sauerstoffionen ausgebaut werden. Details zum Defektmodell sind z. B. in [3] erläutert. In dieser Arbeit werden Sauerstoffpartialdrücke bis ca. $10^{-8}$ bar betrachtet. Sauerstoffdefizite $\delta$ werden mit GI. (3) berechnet.

$$
\delta=\left[\mathrm{V}_{\mathrm{O}}\right] /\left[\mathrm{Pr}_{\mathrm{x}} \mathrm{Ce}_{1-\mathrm{x}} \mathrm{O}_{2}\right]
$$

\section{CTGS basierter resonanter Sensor}

Durch das Anlegen einer Wechselspannung an einen CTGS-Einkristall im y-Schnitt können Dickenscherschwingungen angeregt werden. Dazu sind schlüssellochförmige Platin/Rhodium-Elektroden erforderlich, auf die weitere Sensorschichten abgeschieden werden. Die Schichtabfolgen und das Layout der Schichten sind in Abb. 1 dargestellt.

Die Frequenz am Konduktanzmaximum, d.h. die Serien-Resonanzfrequenz $f\left(G_{\max }\right)=f_{\text {res }}$, wird aufgrund der Unabhängigkeit von Streukapazitäten verfolgt [5]. Das Konduktanzspektrum kann zudem in der Umgebung der Resonanzfrequenz mit einer LorentzFunktion beschrieben werden [6], was zur Datenauswertung genutzt wird. Da die Resonanzfrequenz ebenfalls temperaturabhängig ist $\left(\partial \mathrm{f} / \partial \mathrm{T} \approx-135 \mathrm{~Hz} \mathrm{~K}^{-1}\right.$ für einen $3,7 \mathrm{MHz}-$ Resonator bei $700{ }^{\circ} \mathrm{C}$ ) können bereits geringe Temperaturschwankungen von $0,01 \mathrm{~K}$ das Messsignal überlagern. Aus diesem Grund wird eine interne Temperaturkompensation der Resonanzfrequenz nach [5] angewendet. Die Messung der Resonanzfrequenz verschiedener Obertöne (z. B. Grundton und 3. Oberton) ermöglicht die Eliminierung der Temperaturabhängigkeit. Eine temperaturkompensierte Resonanzfrequenz $f_{\mathrm{IC}}$ wird in GI. (4) definiert [5].

$f_{\text {IC }}\left(m, c_{i}, \ldots\right)$

$=\mathrm{f}_{\operatorname{Res}}^{[1]}\left(T, m, c_{i}, \ldots\right)-\frac{c^{[1]}}{c^{[3]}} \mathrm{f}_{\operatorname{Res}}^{[3]}\left(T, m, c_{i}, \ldots\right)$

$=f_{\operatorname{Res}}^{[1]}\left(m, c_{i}, \ldots\right)-\frac{c^{[1]}}{c^{[3]}} f_{\operatorname{Res}}^{[3]}\left(m, c_{i}, \ldots\right)$

In GI. (4) sind $T$ die Temperatur, $m$ die abgelagerte Masse, $c_{i}$ die Gaskonzentration der Spezies $i$ und $c^{[1]}, c^{[3]}$ die Temperaturkoeffizienten des Grund- und des dritten Obertons. Die Anwendung der Temperaturkompensation erfordert es, dass die Massensensitivität $S$ und die Massenauflösung $R$ ebenfalls angepasst wer- den, siehe GI. (5) und (6).

$$
\begin{aligned}
& S_{I C}=S^{[1]}\left(1-3 c^{[1]} / c^{[3]}\right) \\
& R_{I C}=\Delta f_{\text {Error }} / S_{I C}
\end{aligned}
$$

Für die Massenauflösung $R_{\mathrm{IC}}$ ergibt sich unter Berücksichtigung von Frequenzschwankungen von $5 \mathrm{~Hz}$ und den experimentellen Bedingungen $\left(3,7 \mathrm{MHz}\right.$-Resonator, $\quad 700{ }^{\circ} \mathrm{C}, \quad 5 \mathrm{~mm}$ Schichtdurchmesser) eine Auflösungsgrenze von ca. 0,4 $\mu \mathrm{g}$. Für eine PCO-Schichtmasse von ca. $300 \mu \mathrm{g}$ lassen sich damit Änderungen des Sauerstoffdefizits von $\delta=0,014$ messen .

\section{Mikrowaage-Modus}

Im Mikrowaage-Modus befindet sich die Sensorschicht auf einer größeren Elektrode, wodurch im Wesentlichen Änderungen der mechanischen Eigenschaften der Sensorschicht detektiert werden, d. h. Dichte bzw. Masse. Die Abscheidung einer Masse $\Delta m$ oder die Stöchiometrieänderung einer aufgebrachten dünnen Schicht führen zu einer Resonanzfrequenzänderung $\Delta f$ [4]. GI. (7) kann für Massenänderungen bis zu $2 \%$ der aktiven Resonatormasse angewendet werden, wenn keine anderen Effekte die Resonanzfrequenz beeinflussen.

$\Delta \mathrm{f} / \mathrm{f}=-\Delta \mathrm{m} / \mathrm{m} \rightarrow \Delta \mathrm{m}=-\mathrm{A} \Delta \mathrm{f} / \mathrm{S}$

In Gl. (7) ist $A$ die effektive Resonatorfläche und $S(S=-A \partial f / \partial m)$ die Massensensitivität [5].

\section{Leitfähigkeits-Modus}

Im Leitfähigkeits-Modus überlappt die Sensorschicht die Elektrode, wodurch im Wesentlichen Änderungen der elektrischen Eigenschaften der Sensorschicht detektiert werden. Masseablagerungen auf dem Resonator tragen nur zu einem kleinen Teil zu den Resonanzfrequenzverschiebungen bei. Führen Änderungen der umgebenden Gasatmosphäre zu einer Änderung der elektrischen Leitfähigkeit der Sensorschicht, so verursacht dies eine Veränderung des effektiven Elektrodendurchmessers. Aufgrund der näherungsweise gaußförmigen Verteilung der Massensensitivität (bedingt durch das näherungsweise gaußförmige Schwingungsprofil) verschiebt sich die Resonanzfrequenz, da sich sowohl die effektive Elektroden- und Schichtmasse als auch der effektive piezoelektrische Koeffizient außerhalb der Elektrode ändern.

\section{Experimente}

Einkristalline CTGS-Substrate werden von Fomos-Materials (Russland) bezogen. Die Platin/Rhodium-Elektroden und die PCOSchichten werden mittels gepulster Laserablation auf die CTGS-Substrate aufgebracht. Da- 
für wird ein KrF-Excimer-Laser (248 nm, COMPex 205, Coherent Inc., Santa Clara, USA) verwendet. Das PCO-Target für den Ablationsprozess wird aus stöchiometrisch gemischtem $\mathrm{Pr}_{6} \mathrm{O}_{11^{-}}$(99,9\%, MaTeck $\mathrm{GmbH}$, Deutschland) und $\mathrm{Ce}(\mathrm{IV}) \mathrm{O}_{2}$-Pulver $(99,99 \%$, Chempur $\mathrm{GmbH}$, Deutschland) hergestellt, das zu einer Tablette gepresst (55 MPa) und bei $1350{ }^{\circ} \mathrm{C}$ an Luft gesintert wird.

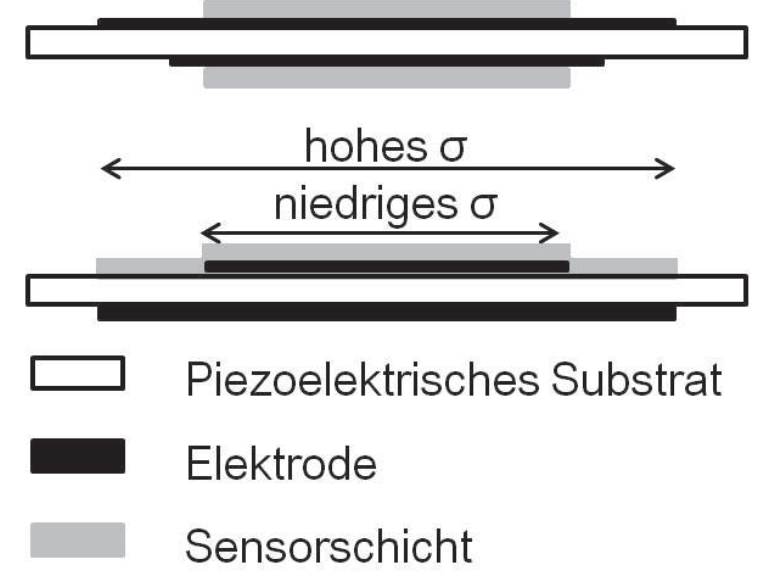

Abb. 1: Schichtabfolge und Layout der Resonatoren für den MikrowaageModus (oben) und den LeitfähigkeitsModus (unten) in der Seitenansicht.

Schichtdicken werden mit einem mechanischen Profilometer XP-2 (Ambios Technology, Inc., USA) bestimmt, indem Höhendifferenzen an den Kanten der Schichten gemessen werden. Die Schichtmassen werden aus der Messung der Resonanzfrequenzverschiebung nach und vor der Schichtabscheidung und der Anwendung von GI. (7) berechnet.

Für Experimente zur Bestimmung des Sauerstoffdefizits von PCO-Schichten wird der Sauerstoffpartialdruck mit Hilfe einer Sauerstoffionen-Pumpe geregelt. Ein Sauerstoffsensor in der unmittelbaren Nähe der Probe ermöglicht die präzise Regelung des Sauerstoffpartialdruckes mit einer Genauigkeit von $\Delta \log \left(\mathrm{p}_{\mathrm{O} 2} /\right.$ bar $)=0,02$. Für eine detaillierte Beschreibung der Sauerstoffionen-Pumpe wird z. B. auf [7] verwiesen. Als Puffergas wird eine Mischung aus Methan-Argon $\left(0,125 \% \mathrm{CH}_{4}\right.$ in $\mathrm{Ar})$ verwendet. Der Gasstrom wird über Massenflussregler (MKS Instruments $\mathrm{GmbH}$, Deutschland) konstant auf $20 \mathrm{~cm}^{3} \mathrm{~min}^{-1}$ geregelt. Die Messung des Sauerstoffdefizits in PCO wird bei $700{ }^{\circ} \mathrm{C}$ in einem Bereich zwischen 0,2 bar und $10^{-8}$ bar durchgeführt. Ein Thermoelement Typ B, das ca. 2 mm unterhalb des Resonators installiert ist, dient zur Messung der Temperatur. Der Resonator befindet sich in einem Probenhalter aus Aluminiumoxid und ist über Platindrähte mit einem Netzwerk- analysator E5100 A (Agilent, USA) verbunden, der die Impedanzspektren in der Nähe der Resonanzfrequenz aufnimmt. Für die Ermittlung der Resonanzfrequenz wird eine Dreipunktkalibrierung (offener Kontakt, Kurzschluss und Widerstand von 47,3 $\Omega$ ) angewendet. Die Resonanzfrequenz kann per Software verfolgt werden. Jedes Resonanzspektrum beinhaltet 400 Datenpunkte. Die Resonanzfrequenzen des Grund- und des dritten Obertons werden unmittelbar nacheinander gemessen. Elektroden- und Sensorschichtdurchmesser entsprechen dem MikrowaageModus und betragen $6 \mathrm{~mm}$ bzw. $5 \mathrm{~mm}$. Abbildung 2 zeigt ein Schema des Versuchsaufbaus.

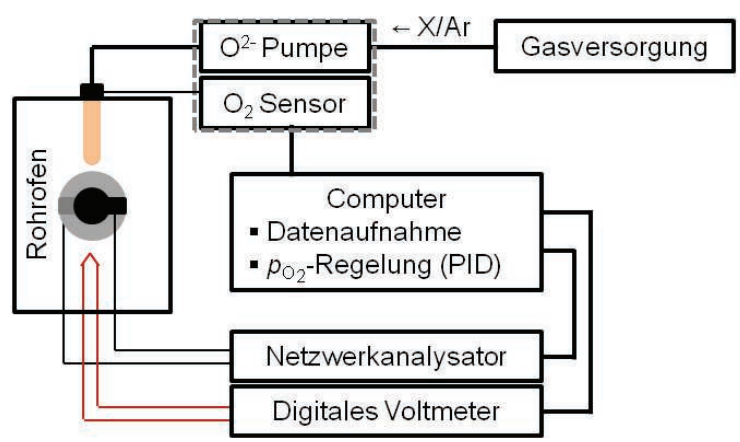

Abb. 2: Messaufbau zur Untersuchung der Sauerstoffpartialdruckabhängigkeit des Sauerstoffdefizits in dünnen PCO-Schichten. Als Puffergas wird eine Mischung aus Argon-Methan verwendet.

Für Experimente zur Bestimmung der durch Änderung der Methankonzentration verursachten Frequenzverschiebungen werden Resonatoren für den Betrieb im Leitfähigkeits-Modus genutzt. Die PCO-Schicht und die untere Elektrode haben einen Durchmesser von $8 \mathrm{~mm}$, die obere Elektrode von $5 \mathrm{~mm}$ (siehe Abb. 1). Die Methankonzentration der Ausgangsgasmischung (5\% Methan in Argon) wird durch ein zweistufiges Gasmischsystem weiter mit Argon verdünnt. Abbildung 3 zeigt das Fließschema des Gasmischsystems. Die Auswirkungen der Konzentrationsänderungen werden bei $700{ }^{\circ} \mathrm{C}$ für Konzentrationen zwischen 125 ppm und 10000 ppm $\mathrm{CH}_{4}$ untersucht.

\section{Ergebnisse}

Die Schichtmassen und -dicken sind in Tabelle 1 zusammengefasst. Für den im Mikrowaage-Modus betriebenen Sensor betragen die Schichtmassen der einzelnen Seiten $77 \mu \mathrm{g}$ bzw. $93 \mu \mathrm{g}$.

\section{Massenänderungen der PCO-Schicht}

Die nominelle Messtemperatur beträgt $700{ }^{\circ} \mathrm{C}$. 
Kleinere Schwankungen werden durch die o. g. Temperaturkompensation ausgeglichen. Die dabei genutzten Temperaturkoeffizienten des $3,7 \mathrm{MHz}$-Resonators bei $700{ }^{\circ} \mathrm{C}$ betragen ca. $136 \mathrm{~Hz} \mathrm{~K}^{-1}$ für den Grundton und $376 \mathrm{~Hz} \mathrm{~K}^{-1}$ für den dritten Oberton. Aus dem Verhältnis der Temperaturkoeffizienten ergibt sich, dass die temperaturkompensierte Massensensitivität nur noch ca. $9 \%$ der Massensensitivität des Grundtons entspricht. Die Verringerung der Massensensitivität wird in Kauf genommen, da temperaturbedingte Schwankungen entfallen.

Tab. 1: Schichtmassen und -dicken

\begin{tabular}{|c|c|c|}
\hline & $\begin{array}{c}\text { Schicht- } \\
\text { masse } \\
{[\mu \mathrm{g}]}\end{array}$ & $\begin{array}{c}\text { Schicht- } \\
\text { dicke } \\
{[\mathrm{nm}]}\end{array}$ \\
\hline $\begin{array}{c}\text { Mikrowaage- } \\
\text { Modus }\end{array}$ & 170 & $1083 \pm 10$ \\
\hline $\begin{array}{c}\text { Leitfähigkeits- } \\
\text { Modus }\end{array}$ & 22 & $182 \pm 18$ \\
\hline
\end{tabular}

Die temperaturkompensierte Resonanzfrequenz an Luft wird als Referenzwert gewählt, woraus sich Frequenz- und Massenänderungen von ca. $-80 \mathrm{~Hz}$ bzw. $11 \mu \mathrm{g}$ ergeben. Das negative Vorzeichen der Frequenzänderung ergibt sich bei der Berechnung der temperaturkompensierten Resonanzfrequenz. Abbildung 4 zeigt die gemessenen Änderungen der temperaturkompensierten Resonanzfrequenz und die daraus mit GI. (7) berechneten Massenänderungen. Bis zu einem Sauerstoffpartialdruck von ca. $10^{-4}$ bar ändert sich die Masse der PCO-Schicht um ca. $9 \mu \mathrm{g}$. Für geringere Sauerstoffpartialdrücke sind die Änderungen kleiner, was dem Defektmodell und Literaturdaten [3] entspricht. Daraus berechnete Sauerstoffdefizite liegen jedoch um ca. den Faktor 7 über den erwarteten Literaturwerten. Eine mögliche Erklärung für diese Abweichung wird in Abschnitt 6 gegeben. Im Sinne der Sensorik ergibt sich daraus keine Einschränkung.

\section{Frequenzänderungen im Leitfähigkeits-Modus}

In Abbildung 5 ist die Verschiebung der temperaturkompensierten Resonanzfrequenz gegen die Methankonzentration aufgetragen. Als Referenz wird auch hier die temperaturkompensierte Resonanzfrequenz an Luft gewählt. Das Einleiten von Methan in den Ofen führt zu einem Sprung in der temperaturkompensierten Resonanzfrequenz von ca. $-60 \mathrm{~Hz}$ für 125 ppm Methan. Bis 1500 ppm ändert sich die temperaturkompensierte Resonanzfrequenz in erster Näherung linear. Für höhere
Konzentrationen bis 10000 ppm schwankt die temperaturkompensierte Resonanzfrequenz um ca. $-165 \mathrm{~Hz}$. Die Messpunkte bei 250 ppm und 3000 ppm werden als Ausreißer betrachtet.

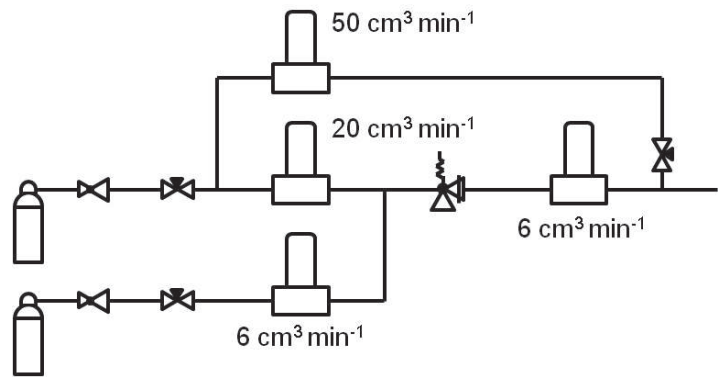

Abb. 3: Zweistufiges Gasmischsystem zur Einstellung von Gaskonzentrationen der Schlüsselgasspezies. Ein Überdruckventil nach der ersten Verdünnung stellt sicher, dass zulässige Druckdifferenzen nicht überschritten werden können.

\section{Diskussion}

Die Abweichungen der Sauerstoffdefizite von den Literaturwerten könnte durch zusätzliche Einflüsse auf die Resonanzfrequenz verursacht werden. In PCO-Schichten treten bei der Einlagerung bzw. dem Ausbau von Sauerstoff durch die chemische Expansion [8] verursachte Spannungen auf, die aufgrund der Unterschiede der Schichtdicke zwischen der Oberund Unterseite der Probe (Massenunterschied: $16 \mu \mathrm{g}) \mathrm{zu}$ einer Biegung des Resonators führen könnten. Eine Biegung hätte zusätzlichen Einfluss auf die Resonanzfrequenz.

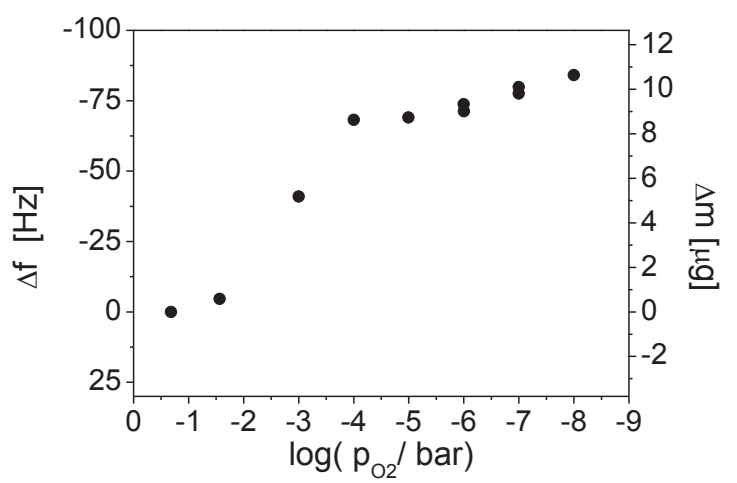

Abb. 4: Gemessene Resonanzfrequenzänderungen der temperaturkompensierten Resonanzfrequenz und mit der Sauerbrey-Gleichung berechnete Massenänderungen in Abhängigkeit des Sauerstoffpartialdruckes bei $700{ }^{\circ} \mathrm{C}$. 


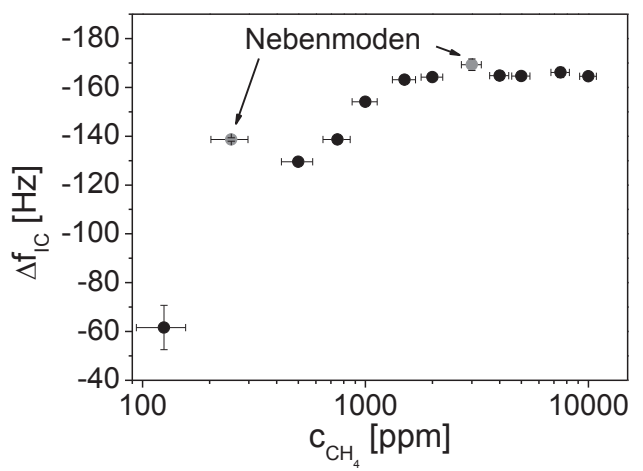

Abb. 5: Temperaturkompensierte Resonanzfrequenz eines Resonators im Leitfähigkeits-Modus in Abhängigkeit der Methankonzentration. Der Fehler für die temperaturkompensierte Resonanzfrequenz entspricht der statistischen Schwankung der Messwerte (1. Konfidenzintervall). Für einige Datenpunkte sind die statistischen Schwankungen geringer als die GröBe der Symbole. Die Unsicherheiten der Konzentrationen resultieren aus den Geräteunsicherheiten der Massenflussregler für die zweistufige Verdünnung der Ausgangsgaskonzentration.

Die Ausreißer der temperaturkompensierten Resonanzfrequenz für den Resonator im Leitfähigkeits-Modus werden sehr wahrscheinlich von einer auftretenden Nebenmode im dritten Oberton verursacht, die sich als Schulter im Konduktanzspektrum manifestiert. Eine nachträgliche Korrektur der temperaturkompensierten Resonanzfrequenz ist mit den gemessenen Daten nicht möglich, da die Lage der Nebenmode nicht eindeutig identifiziert werden kann.

\section{Schlussfolgerungen}

Im Mikrowaage-Modus ist eine Korrelation zwischen der Änderung der temperaturkompensierten Resonanzfrequenz und dem Sauerstoffpartialdruck erkennbar. Die Verbrennung von Methan senkt den Sauerstoffpartialdruck der Ofenatmosphäre, wodurch die PCOSchicht Sauerstoff freisetzt und seine Schichtmasse verringert. Dünne PCO-Schichten könnten somit prinzipiell als Sensorschichten zur Detektion von Methan in der Ofenatmosphäre eingesetzt werden. Eine Reduzierung der Massenunterschiede zwischen der Ober- und Unterseite des Sensors sollte den Einfluss der chemischen Expansion von PCO auf das Messsignals minimieren. Die Korrelation zwischen Sauerstoffpartialdruck und Sauerstoffde- fizit bekräftigt zudem die mögliche Anwendung von PCO als Sensorschicht für den Leitfähigkeits-Modus, da sich mit dem Sauerstoffdefizit auch die elektrische Leitfähigkeit ändert.

Die gemessenen temperaturkompensierten Resonanzfrequenzänderungen des Resonators im Leitfähigkeits-Modus zeigen, dass mit der verwendeten Sensorschicht- und Resonatorgeometrie Änderung der temperaturkompensierten Resonanzfrequenz die Methankonzentrationen zwischen 125 ppm und 1500 ppm widerspiegeln. Der Messbereich kann einerseits durch Variation des Elektroden- und Sensorschichtdurchmessers in Grenzen angepasst werden. Zum anderen könnte die Detektion geringerer Methankonzentrationen mit einer Erhöhung der Sensorschichtdicke erreicht werden [9].

Die Steigerung der Selektivität wird durch die Kombination beider Messmodi erreicht, da die Massenänderungen neben der Einlagerung bzw. dem Ausbau von Sauerstoff auch von den Oberflächenabsorptionsprozessen der jeweiligen Gasspezies abhängen [10]. Die Änderung der elektrischen Eigenschaften der Sensorschicht über den Leitfähigkeits-Modus zu messen, hat dabei den wesentlichen Vorteil, dass die Datenerfassung auf eine hier bereits genutzte Technik, d. h. die Resonanzfrequenzmessung, beschränkt werden kann [10].

\section{Zusammenfassung und Ausblick}

Ein Sensorkonzept, das die Selektivität metalloxid-basierter Gassensoren durch die Messung der mechanischen und elektrischen Eigenschaften der Sensorschicht verbessert, wird zur Detektion von Methan bei $700^{\circ} \mathrm{C}$ eingesetzt. Dazu werden die Sensorschichten auf hochtemperaturstabile, piezoelektrische Resonatoren abgeschieden. Die Unterscheidung der elektrischen und mechanischen Sensorschichteigenschaften wird durch das Layout der Elektroden und Sensorschichten ermöglicht. Einkristalline $\mathrm{Ca}_{3} \mathrm{TaGa}_{5} \mathrm{Si}_{2} \mathrm{O}_{14}-$ Resonatoren ( $y$ Schnitt) dienen als resonante Mikrowaage. Es wird die Tauglichkeit von $\mathrm{Pr}_{0,1} \mathrm{Ce}_{0,9} \mathrm{O}_{2}$-(PCO-)Sensorschichten zur Detektion von Methan bei $700{ }^{\circ} \mathrm{C}$ untersucht. Änderungen der mechanischen Eigenschaften von PCO werden gemessen, indem die PCOSchicht auf eine größere Elektrode aufgebracht wird (Mikrowaage-Modus, siehe Abb. 1 oben). Änderungen des Sauerstoffpartialdruckes korrelieren dann mit Änderungen der temperaturkompensierten Resonanzfrequenz bzw. mit Massenänderungen der PCO-Schicht. Überlappt die PCO-Schicht die Elektrode (Leitfähigkeits-Modus, siehe Abb. 1 unten) können Änderungen der elektrischen Eigenschaften gemessen werden. Die Kombination beider 
Messmodi ermöglicht es, aus der Messung der temperaturkompensierten Resonanzfrequenz Informationen über die Methankonzentration der Ofenatmosphäre zu gewinnen.

Für künftige Arbeiten sollen Resonatoren im Mikrowaage-Modus mit möglichst identischen Schichten auf der Ober- und Unterseite präpariert werden. Es sollen zudem Kennlinienfelder für weitere Schlüsselgasspezies und andere Sensorschichten und Temperaturen vermessen werden. Zur Ausdehnung des Messbereichs von Resonatoren im LeitfähigkeitsModus sollen anderen Elektroden- und Sensorschichtdurchmesser und dickere Sensorschichten erprobt werden. Außerdem sollen weitere Sensorschichtmaterialien zur Detektion weiterer Schlüsselgasspezies und deren Querempfindlichkeiten untersucht werden.

\section{Danksagung}

Die Autoren danken dem Bundesministerium für Wirtschaft und Energie, dem Projektträger Jülich (Förderkennzeichen 03ET1467B) und dem Energie-Forschungszentrum (EFZ, Goslar) für die finanzielle Unterstützung.

\section{Literaturverzeichnis}

[1] P. Quadbeck, A. Strauß, S. Müller, B. Kieback, Atmospheres monitoring in a continuous sintering belt furnace, Journal of Materials Processing Technology 231, 406-411 (2010); doi: 10.1016/j.jmatprotec.2016.01.015

[2] Y. Suhak, M. Schulz, H. Wulfmeier, W. L. Johnson, A. Sotnikov, H. Schmidt, S. Ganschow, D. Klimm, H. Fritze, Langasite-Type Resonant Sensors for Harsh Environments, MRS Advances 1, 1513-1518 (2016); doi: 10.1557/adv.2016.109

[3] S.R. Bishop, T.S. Stefanik, H. L. Tuller, Electrical conductivity and defect equilibria of $\operatorname{Pr}_{0.1} \mathrm{Ce}_{0.9} \mathrm{O}_{2-\delta}$, Physical Chemistry and Chemical Physics 13, 10165-10173 (2011); doi: 10.1039/COCP02920C

[4] G. Sauerbrey, Verwendung von Schwingquarzen zur Wägung dünner Schichten und Mikrowägung, Zeitschrift für Physik 155, 206-222 (1959)

[5] H. Fritze, High-temperature bulk acoustic wave sensors, Measurement Science and Technology 22, 01200-01230 (2011), doi: 10.1088/09570233/22/1/012002

[6] A. Bund, G. Schwitzgebel, Signal-oscillation of a piezoelectric quart crystal in liquids caused by compressional waves, Analytica Chimica Acta 364, 189-194 (1998); doi: 10.1016/S00032670(98)00201-3

[7] M. Schulz, J. Brillo, C. Strenzel and H. Fritze, Oxygen partial pressure control for microgravimetry experiments, Solid State Ionics 225, 332-336 (2012); doi: 10.1016/j.ssi.2012.04.008
[8] J. Seth, D. Chen, J. J. Kim, W.J. Bowman, P. A. Crozier, H. L. Tuller, S. T. Misture, S.

Zdzieszynski, B. W. Sheldon, S. R. Bishop, Coupling of strain, stress, and oxygen nonstoichiometry in thin film $\mathrm{Pr}_{0.1} \mathrm{Ce}_{0.9} \mathrm{O}_{2-\delta}$, Nanoscale 8, 16499-16510 (2016); doi: $10.1039 / \mathrm{c} 6 \mathrm{nr} 04083 \mathrm{~g}$

[9] D. Richter, Selektiver Gassensor auf der Basis hochtemperaturstabiler piezoelektrischer Langasitresonatoren, Dissertation TU Clausthal, S. 6670 (2010)

[10] H. Fritze, D. Richter, H. L. Tuller, Simultaneous detection of atmosphere induced mass and conductivity variations using high temperature resonant sensors, Sensors and Actuators B 111-112, 200-206 (2005); doi: 10.1016/j.snb.2005.06.036 